\section{MS44-O4 Pair Distribution Function calculated from electron diffraction data}

Tatiana E. Gorelik ${ }^{\mathrm{I}}$

1. University of Mainz

email: gorelik@uni-mainz.de

Structure description through Pair Distribution Function (PDF) analysis has attracted particular interest in the last years with the declination of the technology towards nanocrystalline and amorphous materials. The structure of these materials cannot be described by a set of lattice basis vectors and atomic coordinates, but by a continuous function representing the probability to find atoms at a particular distance - PDF. Experimentally, the PDF is calculated from continuous scattering data, as a rule $\mathrm{X}$-ray, or neutron diffraction. The employment of electron diffraction data for the PDF analysis, although not being completely new, survives a renaissance within the last years. Electron diffraction data for the PDF calculation is collected in a Transmission Electron Microscope (TEM), which has to be operated in a special way in order to collect optimal data for PDF. 2D electron diffraction patterns should be integrated into the 1D intensity profile, which then after a proper normalization will be transformed into a PDF. Specifics of electron diffraction data handling as well as the limitation of the method will be discussed. PDFs calculated from electron diffraction data for different materials will be presented and type of structural information extracted from the PDFs will be discussed.

Keywords: Total scattering, electron diffraction, pair-distribution function

\section{MS44-O5 Searching for Ti-clusters in $\mathrm{Mg}_{0.7} \mathrm{Ti}_{0.3}$ thin film}

Hyunjeong $\mathrm{Kim}^{1}$, Kohta Asano ${ }^{1}$, Kouji Sakaki ${ }^{1}$, Yumiko Nakamura $^{1}$, Akihiko Machida ${ }^{2}$, Naoyuki Maejima ${ }^{2}$, Tetsu Watanuki ${ }^{2}$, Herman Schreuders ${ }^{3}$, Bernard Dam ${ }^{3}$

1. National Institute of Advanced Industrial Science and Technology, Tsukuba, Ibaraki 305-8565, Japan

2. National Institutes for Quantum and Radiological Science and Technology, Sayo, Hyogo 679-5148, Japan

3. Delft University of Technology, Julianalaan 136, NL-2628 BL Delft, The Netherlands

email: hj.kim@aist.go.jp

Mg-rich $\mathrm{Mg}_{1-\mathrm{Ti}}$ thin film absorbs a large amount of hydrogen in ambient conditions. When in the hydride phase, it absorbs $\sim 87 \%$ of solar radiation with low thermal emissivity $[1,2]$. This makes $\mathrm{Mg}$-rich $\mathrm{Mg}_{1-\mathrm{T}} \mathrm{Ti}$ thin film very attractive for a switchable smart coating material for solar collectors. Lattice parameters of

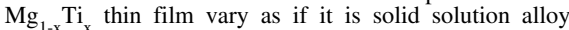
following Vegard's law [2]. However, their optical and electrical properties are not well explained by such a simple structural model. Indeed, a more complex model like nanosized Ti-clusters embedded in Mg-matrix is required [2]. However, there has been no clear evidence for the existence of such nano-clusters in this material.

To resolve Ti-clusters, we obtained the X-ray atomic pair distribution functions (PDFs) of $\mathrm{Mg}_{0.7} \mathrm{Ti}_{0.3}$ thin film during the hydrogenation process at $\mathrm{BL} 22 \mathrm{XU}$ at SPring-8. The PDF before hydrogenation is well explained by a hexagonal close packed (hcp) structural model over a wide interatomic distance range. At the early stage of hydrogenation, a part of the PDF is gradually transformed into a face centered cubic (fcc) structure. Change occurs only in the low- $r$ region and the high- $r$ region of the PDF stays in an hep structure. Such change in the PDF is expected: it has been proposed that when the $\mathrm{Mg}_{0.7} \mathrm{Ti}_{03}$ thin film is hydrogenated, Ti clusters absorb hydrogen first transformed into an fcc structure while the Mg-matrix stays in a hcp structure [2]. The Mg-matrix absorbs hydrogen later. Our PDF study suggests that the average size of Ti-clusters in $\mathrm{Mg}_{0.7} \mathrm{Ti}_{0.3}$ thin film is $\sim 30 \AA$.

This work is partly supported by Photon and Quantum Basic Research Coordinated Development Program from the Ministry of Education, Culture, Sports, Science and Technology, Japan.

[1] D. M. Borsa et al., Appl. Phys. Lett. 88, 241910 (2006).

[2] D. M. Borsa et al., Phys. Rev. B 75, 205408 (2007).

Keywords: nano-clusters, thin film, $\mathrm{x}$-ray atomic pair distribution function 